\title{
Semimartingale Property and Its Connections to Arbitrage*
}

\author{
Sallieu Kabay Samura, Junjun Mao, Dengbao Yao \\ School of Mathematical Science, Anhui University, Hefei, China \\ Email: ssallieu@yahoo.com,maojunjun@ahu.edu.cn,yaodengbao@126.com
}

Received January 27, 2013; revised March 25, 2013; accepted April 11, 2013

Copyright (C) 2013 Sallieu Kabay Samura et al. This is an open access article distributed under the Creative Commons Attribution License, which permits unrestricted use, distribution, and reproduction in any medium, provided the original work is properly cited.

\begin{abstract}
In this paper, we prove the celebrated Bichteler-Dellaccherie Theorem which states that the class of stochastic processes $X$ allowing for a useful integration theory consists precisely of those processes which can be written in the form $X=X_{0}$ $+M+A$, where $M_{0}=A_{0}=0, M$ is a local martingale, and $A$ is of finite variation process. We obtain this decomposition rather direct form an elementary discrete-time Doob-Meyer decomposition. By moving to convex combination we obtain a direct continuous time decomposition, which then yield the desired decomposition. We also obtain a characterization of semi-martingales in terms of a variant no free lunch with vanishing risk.
\end{abstract}

Keywords: Bichteler-Dellaccherie Theorem; Doob-Meyer Decomposition; Semi-Martingales; Arbitrage; Komlos Lemma

\section{Introduction}

In this paper, $\left(\Omega, \mathbb{F},\left(\mathbb{F}_{t}\right)_{t \in \mathbb{R}_{+}}, P\right)$ is assumed to be a filtered probability space where $\left(\mathbb{F}_{t}\right)_{t \in \mathbb{R}_{+}}$is a filtration satisfying $\mathbb{F}_{t} \subseteq \mathbb{F}$ for all $t \in \mathbb{R}_{+}$, the usual condition of right continuity and completeness. The random movement of $d \in \mathbb{N}$ risky assets in the market is modeled via cadlag, nonnegative stochastic processes $X_{i}$, where $i \in\{1, \cdots, d\}$. We assume that all wealth processes are discounted by another special asset which is considered a baseline. In the market described above, economic agents can trade in order to reallocate their wealth.

Consider a simple predictable process

$$
\phi=\sum_{j=0}^{n-1} \eta_{j} \mathbb{I}_{\left|\tau_{j-1}, \tau_{j}\right|} .
$$

where $\tau_{0}=0$, and for all $j \in\{1, \cdots, n\}, \tau_{j}$ is a finite stopping time and $\eta_{j}=\left(\phi_{j}^{i}\right)_{i=1, \cdots, d}$ is $\mathbb{F}_{\tau_{j-1}}$-measurable. Each $\tau_{j-1}, \quad j \in\{1, \cdots, n\}$, is an instance when some give economic agent may trade in the market, then, $\eta_{j}^{i}$ is the number of unit from the ith risky assets that the agent will hold in the trading interval $\left[\tau_{j-1}, \tau_{j}\right]$. This form of trading is called simple, as it comprises of finite number

\footnotetext{
*Supported by the NNSF of China (11171001), HSSPF of the Ministry of Education of China (10YJA910005), Talents youth Fund of Anhui Province Universities (2010SQRL016ZD), Academic Innovation Team of Anhui University (KJTD001B) and Youth Science Research Fund of Anhui University (2009QN011A).
}

of buy-and-hold strategies, in contrast to continuous trading where one is able to change the position of the assets in a continuous fashion. The last form of trading is only theoretical value, since it cannot be implemented in reality, even if one ignores market frictions.

Starting from initial capital $x \in \mathbb{R}_{+}$and following the strategy described by the simple predictable process $\phi=\sum_{j=0}^{n-1} \eta_{j} \mathbb{I}_{\left|\tau_{j-1}, \tau_{j}\right|}$, the agent's discounted process is given by

$$
X^{x, \phi}=x+\sum_{j=0}^{n-1} \eta_{j+1}\left(X_{t \Lambda \tau_{j+1}}-X_{t \Lambda \tau_{j}}\right) .
$$

where $n \in \mathbb{N}, 0=\tau_{0} \leq \tau \leq \cdots \leq \tau_{n}$ are a.s. finite stoping times with respect to $\mathbb{F}_{t}$ and the $\phi_{j}$ are $\mathbb{F}_{\tau_{j}}$-measurable real random variables. Note that the trader is allowed to trade on an infinite time horizon, because we do not restrict to bounded stoping times for the re-allocation of the capital. Of course trading on a finite time horizon $[0$, $T]$ is covered by switching to the process $\left(X_{t \Lambda T}, \mathbb{F}_{t \Lambda T}\right)$.

Theorem 1.1. [1,2] A real valued, cadlag, adapted process $X=\left(X_{t}\right)_{0 \leq t \leq T}$ the following are equivalent:

1) $X$ is a good integrator.

2) $X$ may be decomposed as $X=M+A$, where $M=\left(M_{t}\right)_{0 \leq t \leq T}$ is a local martingale and $A=\left(A_{t}\right)_{0 \leq t \leq T}$ is an adapted process of finite variation.

Defination 1.1. [1,3] A real valued, cadlag, adapted 
process $X=\left(X_{t}\right)_{0 \leq t \leq T}$ allows for A Free Lunch With Vanishing Risk for simple integrands if there is a sequence $\left(\phi^{n}\right)_{n=1}^{\infty}$ of simple integrands such that for $n \rightarrow \infty$,

$$
\left(\phi^{n} \cdot X\right)_{T}^{+} \nrightarrow 0 \text { in probability . }
$$

and

$$
\sup _{0 \leq t \leq T}\left\|\left(\phi^{n} \cdot X\right)_{t}^{-}\right\|_{\infty}=\left\|\left(\phi^{n} \cdot X\right)^{-}\right\| \rightarrow 0
$$

In contrast, $X$ therefore admits No Free Lunch With Vanishing Risk (NFLVR) for simple integrands if for every sequence $\left(\phi^{n}\right)_{n=1}^{\infty} \in S I$ satisfying (VR) we have

(NFL) $\quad\left(\phi^{n} \cdot X\right)_{T} \rightarrow 0$ in probability.

A free lunch with vanishing risk (FLVR) for simple integrands indicates that $\mathrm{S}$ allows for a sequence of trading schemes $\left(\phi^{n} \cdot X\right)_{n=1}^{\infty}$, each $\phi^{n}$ involving only finitely many rebalancing of the portfolio, such that the losses tend to Zero in the sense that of (VR), while the terminal gains (FL) remain substantial as $\mathrm{n}$ goes to infinity. It is important to note that the condition (VR) of vanishing risk pertains the maximal losses of the trading strategy $\phi^{n}$ during the entire interval $[0, T]$ : if the left hand side of (VR) equals $\varepsilon_{n}$ this implies that, with probability one, the strategy $\phi^{n}$ never, i.e. for not $t \in[0, T]$, cause an accumulated loss of more than $\varepsilon_{n}$.

Resently, it has been argued that existence of an Equivalent Martingale Measure(EMM) is not necessary for viability of the market; to see this effect, see [4-6]. In [7], the concept of strictly positive supermartingale deflator which is weaker than the existence of an EMM, that allows for consistent theory to be developed.In this paper, we investigate the relation between the no free lunch with vanishing risk property for simple integands and the semimartingale property.

Theorem 1.2. [1,8] Let $\left(X_{t}\right)_{0 \leq t \leq T}$ be a real-valued, cadlag, locally bounded process based on and adepted to a filtered probability space $\left(\Omega, \mathbb{F},(\mathbb{F})_{0 \leq t \leq T}, \mathbb{P}\right)$. If $S$ satisfies the condition of no free lunch with vanishing risk (NFLVR) for simple integrands then $\mathrm{S}$ is a semimartingale.

Theorem 1.3. For a locally bounded, adopted, cadlag process $X$ the following are equivalent

1) $X$ satisfies NFLVR + LI(little Investment)

2) $X$ is a classical semimartingale.

Theorem 1.4. For an adapted cadlag process $X$ the following are equivalent.

1) For all sequences $\left(\phi^{n}\right)_{n \geq 1}$ of simple predictable processes,

a) $\lim \left\|\phi^{n}\right\|_{\infty}=0$ b) $\lim _{n \rightarrow \infty} \sup _{0 \leq t \leq T}\left(\phi^{n} \cdot X\right)_{t}^{-}=0$ together imply $\left(\phi^{n} \cdot X\right)_{t}^{-} \rightarrow 0$ in probability.

2) $X$ is a classical semimartingale.

Proposition 1.5. Let $X=\left(X_{t}\right)_{0 \leq t \leq 1}$ be cadlag and adapted, with $X_{0}$ and such that $\|X\| \leq 1$ and $X$ satisfies NFLVR + LI For all $\epsilon>0$ there is $C>0$ and a sequence of stopping times $\left(\tau_{n}\right)_{n \geqslant 1}$ such that, for all $n$

1) $\tau_{n}$ takes values in $D_{n} \cup\{\infty\}$.

2) $P\left(\tau_{n}<\infty\right)<\varepsilon$.

3) The stopped processes $A^{n, \tau_{n}}$ and $M^{n, \tau_{n}}$ satisfy, for all $n,\left\|M_{1}^{n, \tau_{n}}\right\|_{L^{2}}^{2} \leq C$ and

$$
\operatorname{TV}\left(A^{n, \tau_{n}}\right)=\sum_{j=1}^{2^{n}}\left|A_{j 2^{-n}}^{n, \tau_{n}}-A_{(j-1)^{-n}}^{n, \tau_{n}}\right| \leq C
$$

Lemma 1.6. Under the assumptions as in the proposition above with

$$
Q^{n}=\sum_{j=1}^{2^{n}}\left(X_{j 2^{-n}}-X_{(j-1)^{2-n}}\right)^{2},
$$

the sequence $\left(Q^{n}\right)_{n \geq 1}$ is bounded in probability.

Proof. For all $n$, let

$$
\phi^{n}=-\sum_{j=1}^{2^{n}} X_{(j-1) 2^{-n}} I\left[(j-1) 2^{-n}, j 2^{n}\right]
$$

a simple predictable process, then $\left\|\phi^{n}\right\|_{\infty} \leq 1$ since $\|X\|_{\infty} \leq 1$

$$
\begin{gathered}
\left(\phi^{n} \cdot X\right)_{t}=-\sum_{j=1}^{2^{n}} X_{t \Lambda(j-1) 2^{n}}\left(X_{t \Lambda j 2^{-n}}-X_{t \Lambda(j-1) 2^{-n}}\right) \\
=\frac{1}{2} \sum_{j=1}^{2^{n}}\left(X_{t \Lambda j 2^{-n}}-X_{t \Lambda(j-1) 2^{-n}}\right)^{2}+\frac{1}{2}\left(X_{0}^{2}-X_{t}^{2}\right) \geq-\frac{1}{2} . \\
t=1 \\
\left(\phi^{n} \cdot X\right)_{1}=\frac{1}{2} Q^{n}+\frac{1}{2}\left(X_{0}^{1}-X_{t}^{2}\right)
\end{gathered}
$$

since $X$ satisfies NFLVR $+\mathrm{LI},\left(\left(\phi^{n} \cdot X\right)_{1}, n \geq 1\right)$ is bounded in $L^{0}(P)$.

For $c>0$ define a sequence of stopping times

$$
\sigma_{n}(c)=\inf \left\{\frac{k}{2^{n}}: \sum_{j=1}^{k}\left(X_{j 2^{-n}}-X_{(j-1)^{2-n}}\right)^{2} \geq c-4\right\} .
$$

Given $\varepsilon \geq 0$ there is $c_{1}$ such that

$$
P\left[\sigma_{n}\left(c_{1}\right)<\infty\right]<\frac{\varepsilon}{2}
$$

Lemma 1.7. Under the same assumptions as in Proposition 1.5 the stopped martingales $M^{n, \sigma_{n}\left(c_{1}\right)}$ satisfy $\left\|M_{1}^{n, \sigma_{n}\left(c_{1}\right)}\right\|_{L^{2}}^{2} \leq C_{1}$.

Proof. For $n \geq 1$ and $k=1, \cdots, 2^{n}$, since the $A^{n} s$ are predictable and the $M^{n} s$ are martingales, 


$$
\begin{aligned}
& E\left[\left(X_{k 2^{-n}}^{\sigma_{n}\left(c_{1}\right)}-X_{(k-1) 2^{-n}}^{\sigma_{n}\left(c_{1}\right)}\right)^{2}\right] \\
& =E\left[\left(M_{k 2^{-n}}^{\sigma_{n}\left(c_{1}\right)}-M_{(k-1) 2^{-n}}^{\sigma_{n}\left(c_{1}\right)}\right)^{2}\right]+E\left[\left(A_{k 2^{-n}}^{\sigma_{n}\left(c_{1}\right)}-A_{(k-1) 2^{-n}}^{\sigma_{n}\left(c_{1}\right)}\right)^{2}\right] \\
& \geq E\left[\left(M_{k 2^{-n}}^{\sigma_{n}\left(c_{1}\right)}\right)^{2}-\left(M_{(k-1) 2^{-n}}^{\sigma_{n}\left(c_{1}\right)}\right)^{2}\right]
\end{aligned}
$$

we write $E\left[\left(M_{1}^{\sigma_{n}\left(c_{1}\right)}\right)^{2}\right]$ as a telescoping series and simplifying to get

$$
\begin{aligned}
& E\left[\left(M_{1}^{\sigma_{n}\left(c_{1}\right)}\right)^{2}\right] \\
& =\sum_{k 2^{-n} \leq \sigma_{n}\left(c_{1}\right)} E\left[\left(X_{k 2^{-n}}^{\sigma_{n}\left(c_{1}\right)}-X_{(k-1) 2^{-n}}^{\sigma_{n}\left(c_{1}\right)}\right)^{2}\right] \\
& +E\left[\left(X_{\sigma_{n}\left(c_{1}\right)}-X_{\sigma_{n}\left(c_{1} 2^{-n}\right.}\right)^{2}\right] \\
& \leq\left(c_{1}-4\right)+2^{2}=c_{1}
\end{aligned}
$$

Lemma 1.8. Let

$$
V^{n}=T V\left(A^{n, \sigma_{n}\left(c_{1}\right)}\right)=\sum_{i=1}^{2^{n}\left(\sigma_{n}\left(c_{1}\right) \wedge 1\right)}\left|A_{j 2^{-n}}^{n}-A_{(j-1) 2^{-n}}^{n}\right| .
$$

Under the assumption of Proposition 1.5 the sequence $\left(V^{n}\right)_{n>1}$ is bounded in probability.

Proof. Assume for contradiction that $\left(v^{n}\right)_{n>1}$ is not bounded in probability. Then there is $\alpha>0$ such that for all $k$ there is $n_{k}$ such that $p\left[V^{n_{k}}\right] \geq k \geq \alpha$. For $n \geq 1$ define

$$
b_{j-1}^{n}=\operatorname{sign}\left(A_{j 2^{-n}}^{n, \sigma_{n}\left(c_{1}\right)}-A_{(j-1) 2^{-n}}^{n, \sigma_{n}\left(c_{1}\right)}\right) \in \mathbb{F}_{(j-1) 2^{-n}}
$$

and

$$
\phi^{n}(t)=\sum_{j=1}^{2^{n}} b_{j-1}^{n} 1_{\left[(j-1) 2^{-n}, j 2^{-n}\right]}(t) .
$$

Then $\left\|\phi^{n}\right\|_{u} \leq 1$ and

$$
\begin{aligned}
& \left(\phi^{n, \sigma_{n}\left(c_{1}\right)} \cdot X\right)_{t} \\
& =\sum_{j \leq\left[t 2^{n}\right.} b_{j 2^{-n}}^{n}\left(X_{j 2^{-n}}^{\sigma_{n}\left(c_{1}\right)}-X_{(j-1) 2^{-n}}^{\sigma_{n}\left(c_{1}\right)}\right) \\
& \quad+b_{\left[t 2^{n}\right]}\left(X_{t}^{\sigma_{n}\left(c_{1}\right)}-X_{t 2^{-n}}^{\sigma_{n}\left(c_{1}\right)}\right) \\
& \geq\left(\phi^{n}, \sigma_{n\left(c_{1}\right)} \cdot A^{n}\right)_{\left[2^{n}\right]^{-n}}+\left(\phi^{n}, \sigma_{n\left(c_{1}\right)} \cdot M^{n}\right)_{\left[t 2^{n}\right] 2^{-n}}-2
\end{aligned}
$$

and at time $t=1$ we have

$$
\left(\phi^{n, \sigma_{n}\left(c_{1}\right)} \cdot X\right)_{1}=V^{n}+\left(\phi^{n, \sigma_{n}\left(c_{1}\right)} \cdot M^{n}\right)_{1} .
$$

But the second summand is bounded in $L^{2}$, so we conclude that $\left(\phi^{n, \sigma_{n}\left(c_{1}\right)} \cdot X\right)$ is not bounded in probability.
We defined a sequence of stopping times

$$
\eta_{n}(c)=\inf \left\{\frac{j}{2^{n}}: \mid\left(\phi^{n, \sigma_{n}\left(c_{1}\right)} \cdot M^{n}\right)_{j 2^{-n}} \geq c\right\} .
$$

Because

$$
E\left[\left(\sup _{1 \leq j \leq 2^{n}}\left(\left(h^{n, \sigma_{n}\left(c_{1}\right)} \cdot M^{n}\right)_{j 2^{-n}}\right)^{2}\right] \leq 4 c_{1}\right.
$$

by Doob's sub-martingale in-equality,(see $[9,10])$ $\left(\phi^{n, \sigma_{n}\left(c_{1}\right)} \cdot M^{n}\right)$ is bounded in probability. Therefore there is $c^{\prime}>0$ such that $P\left[\eta_{n}\left(c^{\prime}\right)<\infty\right] \leq \alpha / 2$. Note that $\phi^{n, \sigma_{n}\left(c_{1}\right) \Lambda \eta_{n}\left(c^{\prime}\right)} \cdot X$ is uniformly bounded below by $c^{\prime}$. We claim $\left(\phi^{n, \sigma_{n}\left(q_{1}\right) \wedge \eta_{n}\left(c^{\prime}\right)} \cdot X\right)$ is not bounded in probability. Indeed, for any $\mathrm{n}$ and any $\mathrm{k}$,

$$
\begin{aligned}
\alpha & \leq p\left[\left(\phi^{n, \sigma_{n}\left(c_{1}\right) \Lambda \eta_{n}\left(c^{\prime}\right)} \cdot X\right)_{1} \geq k\right] \\
& \leq p\left[\left(\phi^{n, \sigma_{n}\left(c_{1}\right)} \cdot X\right)_{1} \geq k, \eta_{n}\left(c^{\prime}\right)=\infty\right]+P\left[\eta_{n}\left(c^{\prime}\right)<\infty\right] .
\end{aligned}
$$

Since $P\left[\eta_{n}\left(c^{\prime}\right)<\infty\right] \leq \alpha / 2$, the probability of the other event is at least $\alpha / 2$. This gives the desired contradiction because it is now easy to construct a FLVR + LI.

Proof of Proposition 1.5: Defined a sequence of stopping times

$$
\tau_{n}(c):=\inf \left\{\frac{k}{2^{n}}: \sum_{j=1}^{k}\left|A_{j 2^{-n}}-A_{(j-1) 2^{-n}}\right| \geq c\right\} .
$$

By Lemma 1.8 there is $c_{2}$ such that $P\left[\tau_{n}\left(c_{2}\right)<\infty\right]<\varepsilon / 2$. Take $C:=c_{1} \vee c_{2}$ and $\rho_{n}=\sigma_{n}\left(c_{1}\right) \wedge \tau_{n}\left(c_{2}\right)$.

Lemma 1.9. [11]. Let $f \cdot g:[0,1] \rightarrow \Re$ be measurable functions, where $f$ is left continuous and takes finitely many values. Say $f=\sum_{k=1}^{k} f\left(X_{k}\right) 1_{\left(X_{k-1}, s_{k}\right]}$. Define

$$
\begin{aligned}
(f \cdot g)= & \sum_{k=1}^{k} f\left(X_{k-1}\right)\left(g\left(X_{k}\right)-g\left(X_{k-1}\right)\right) \\
& +f\left(X_{k(t)}\right)\left(g(t)-g\left(X_{k(t)}\right)\right)
\end{aligned}
$$

where $k(t)$ is the biggest of the $k$ such that $X_{k}$ less than or equal to $t$. Then for all partition $0 \leq t_{0} \leq \cdots \leq t_{M} \leq 1$,

$$
\begin{aligned}
& \sum_{i=1}^{M}\left|(f \cdot g)\left(t_{i}\right)-(f \cdot g)\left(t_{i-1}\right)\right| \\
& \leq 2 T V(f)\|g\|_{\infty}+\left(\sum_{i=1}^{M}\left|g\left(t_{i}\right)-g\left(t_{i-1}\right)\right|\right)\|f\|_{\infty} .
\end{aligned}
$$

Proposition 2.0. Let $X=\left(X_{t}\right)_{0 \leq t \leq 1}$ be cadlag and adopted, with $X_{0}=0$ and such that $\|X\|_{u} \leq 1$ and $X$ satisfies NFLVR + LI. For all $\varepsilon>0$ there is $C$ and a $[0,1] \cup\{\infty\}$ valued stopping time $\alpha$ such that $p[\alpha<\infty]<\varepsilon$ and sequence $\left(M^{n}\right)_{n \geq 1}$ and $\left(A^{n}\right)_{n \geq 1}$ of continuous time cadlag processes such that for all $n$,
1) $A_{0}^{n}=M_{0}^{n}=0$
2) $X^{\alpha}=A^{n, \alpha}+M^{n, \alpha}$ 
3) $M^{n, \alpha}$ is a martingale with $\left\|M_{1}^{n, \alpha}\right\|_{L^{2}}^{2} \leq C$

4) $\sum_{j=1}^{2^{n}}\left\|A_{j 2^{-n}}^{n, \alpha}-A_{(j-1) 2^{-n}}\right\| \leq C$

Proof. Let $\varepsilon \geq 0$ be given. Let $C, M^{n}, A^{n}$, and $\rho_{n}$ be as in proposition 1.5. Extended $M^{n}$ and $A^{n}$ to all $t \in[0,1]$ by defining $M_{t}^{n}=E\left[M_{1}^{n} \mid F_{t}\right]$ and $A^{n}=X_{t}-M_{t}$. Not that the extended $A^{n}$ is no longer predictable, and currently we only have control of the total variation of $A^{n, \rho_{n}}$ over $D_{n}$, i.e.

$$
\sum_{j=1}^{2^{n}\left(\rho_{n} \Lambda 1\right)}\left|A_{j 2^{-n}}^{n}-A_{(j-1) 2^{-n}}\right| \leq C .
$$

Notice that, for $t \in\left[(j-1) 2^{-n}, j 2^{-n}\right]$,

$$
\begin{aligned}
A^{n} & =X_{t}-M_{t}^{n}=X_{t}-E\left[M_{j 2^{-n}}^{n} \mid F_{t}\right] \\
& =X_{t}-E\left[X_{j 2^{-n}}^{n}-A_{j 2^{-n}}^{n} \mid F_{t}\right] \\
& =A_{j 2^{-n}}^{n}-\left(E\left[X_{j 2^{-n}}^{n} \mid F_{t}\right]-X_{t}\right)
\end{aligned}
$$

From this and $\|X\|_{u} \leq 1$ it follow that $\left\|A_{t}^{n}-A_{j 2^{-n}}^{n}\right\|_{\infty} \leq 2$, so $\left\|A^{n, \rho_{n}}\right\|_{u} \leq C+2$. How do we fine the limit of the sequence of stopping times $\left(\rho_{n}\right)_{n \geq 1}$ ? The trick is to define $R^{n}=1_{\left[0, \rho_{n} \Lambda 1\right]}$, a simple predicator process, and note that stopping at $\rho_{n}$ is like integrating $R_{n}$, i.e. $A^{n, \rho_{n}}=R^{n} \cdot A^{n}$ and $M^{n, \rho_{n}}=R^{n} \cdot M^{n}$. We have that

$$
1 \geq E\left[R_{1}^{n}\right]=E\left[1_{\rho n}=\infty\right]=1-P\left[\rho_{n} \leq \infty\right] \geq 1-\varepsilon .
$$

Apply Komlos' Lemma to obtain convex weights $\left(\mu_{n}^{n}, \cdots, \mu_{N_{n}}^{n}\right)$ such that

$$
R^{n}=\sum_{i=n}^{\infty} \mu_{i}^{n} R_{1}^{i} \rightarrow R_{1}
$$

a.s as $n \rightarrow \infty$ By the dominated convergence theorem, $E\left[R_{1}\right] \geq 1-\varepsilon$. Observe that

$$
R^{n} \cdot X=\sum_{i=n}^{\infty} \mu_{i}^{n}\left(R_{1}^{i} \cdot M^{i}\right)+\sum_{i=n}^{\infty} \mu_{i}^{n}\left(R_{1}^{i} \cdot A^{i}\right)
$$

Define $\alpha_{n}=\inf \left\{t: R_{t}^{n} \leq 1 / 2\right\}$. Each $R^{n}$ is left continuous, decreasing process. In particular, $R_{\alpha_{n}} \geq 1 / 2>0$, so we can divide by this quantity. We claim that $P\left[\alpha_{n}<\infty\right]<\varepsilon$. In deed, on the event $\left[\alpha_{n}<\infty\right], R_{1}^{n} \leq$ $R_{\alpha_{n}+}^{n} \leq 1 / 2$ so

$$
\begin{aligned}
R_{1}^{n} & \leq \varepsilon \geq E\left[1-R_{1}^{n}\right] \geq E\left[\left(1-R_{\alpha_{n}}^{n}\right) 1_{\alpha<\infty}\right] \\
& \geq 1 / 2 P\left[\alpha_{n}<\infty\right] .
\end{aligned}
$$

Define new processes $T_{t}^{n}=1_{\left[0, \alpha_{n}\right]}(t) / R_{t}^{n}$. Then $\left\|T^{n}\right\|_{n} \leq 2$ and $T^{n} \cdot\left(R^{n} \cdot X\right)=X^{\alpha_{n}}$. Thus we define $M^{n}$ and $A^{n}$ by

$$
\begin{aligned}
X^{\alpha_{n}} & =T^{n} \cdot\left(\sum_{i=n}^{\infty} \mu_{i}^{n}\left(R^{i} \cdot M^{i}\right)\right)+T^{n} \cdot\left(\sum_{i=n}^{\infty} \mu_{i}^{n}\left(R^{i} \cdot A^{i}\right)\right) \\
& =M^{n}+A^{n} .
\end{aligned}
$$

The total variation of $T^{n}$ over $D_{n}$ is bounded by 3 . By Lemma 1.9,

$$
\begin{aligned}
\sum_{j=1}^{2^{n}}\left|A_{j 2^{-n}}-A_{(j-1)}^{n}\right| \leq & 2 T V_{n}\left(T^{n}\right)\left\|\sum_{i=n}^{\infty} \mu_{i}^{n}\left(R^{i} \cdot A^{i}\right)\right\|_{\infty} \\
& +\left\|T^{n}\right\|_{\infty} T V_{n}\left(\sum_{i=n}^{\infty} \mu_{i}^{n}\left(R^{i} \cdot A^{i}\right)\right) \\
\leq & 6(C+2)+2 C
\end{aligned}
$$

That $\left\|M_{1}^{n}\right\|_{L^{2}}^{2} \leq C$ follows from the fact that $\left\|M_{1}^{n, \alpha_{n}}\right\|_{L^{2}}^{2} \leq C$. To finish the proof, we show that there is a subsequence $\left(\alpha_{n_{k}}\right)$ such that $\alpha=\inf _{k} \alpha_{n_{k}}$ satisfies $P[\alpha<\infty] \leq 4 \varepsilon$. We know $P\left[R_{1} \leq 2 / 3\right] \leq 3 \varepsilon$ because $E\left[R_{1}\right] \geq 1-\varepsilon$. Since $R_{1}^{n} \rightarrow R_{1}$ a.s there is a subsequence such that $P\left[\left|R_{1}^{n}-R_{1}\right| \geq 1 / 15\right] \leq \varepsilon 2-k$. Finally,

$$
\begin{aligned}
P[\alpha<\infty] & \leq P\left[\inf _{k} R_{1}^{n_{k}} \leq 2 / 3\right] \\
& \leq 3 \varepsilon+P\left[\inf _{k} R_{1}^{n_{k}} \leq 3 / 5, R_{1}>2 / 3\right] \\
& \leq 3 \varepsilon+\sum_{k=1}^{\infty} P\left[R_{1}^{n_{k}} \leq 3 / 5, R_{1}>2 / 3\right] \\
& \leq 3 \varepsilon+\sum_{k=1}^{\infty} P\left[\left\|R_{1}^{n_{k}}-R_{1}\right\| \leq 1 / 15\right] \\
& \leq 4 \varepsilon
\end{aligned}
$$

Therefore $\left(M^{n}\right)_{n \geq 1},\left(A^{n}\right)_{n \geq 1}$ and $\alpha$ have the desired properties.

Proof of the Main Theorems

Proof of Theorem 1.3. We may assume the hypothesis of proposition. Let $\varepsilon>0$ and take $C, \alpha,\left(M^{n}\right)_{n \geq 1}$, $\left(A^{n}\right)_{n \geq 1}$ as in proposition. Apply komlos lemma to find convex weights $\left(\lambda_{n}^{n}, \cdots, \lambda_{N_{n}}^{n}\right)$ such that

$$
\begin{gathered}
\lambda_{n}^{n} M_{1}^{n, \alpha}+\cdots+\lambda_{N_{n}}^{n} M_{1}^{N, \alpha} \rightarrow M_{1} \\
\lambda_{n}^{n} A_{t}^{n, \alpha}+\cdots+\lambda_{N_{n}}^{n} A_{t}^{N, \alpha} \rightarrow A_{t}
\end{gathered}
$$

for all $t$, where the convergence is a.s. For all $n$, $\sum_{j=1}^{2^{n}}\left|A_{j 2^{-n}}^{n, \alpha}-A_{(j-1) 2^{-n}}\right| \leq C$ so the total variation of $A$ over $D$ is bounded by $C$. Further, we have $X^{\alpha}=M_{t}+A_{t}$. $A$ is a cadlag on $D$, so define it on all of $[0,1]$ to make it cadlag. $M$ is $L^{2}$ martingale so it has a cadlag modification. Since $P[\alpha<\infty]<\varepsilon$ and $\varepsilon>0$ was arbitrary, and the class of classical semimartingales is local, $X$ must be a classical semimartingale.

Proof of Theorem 1.4. We no longer assume that $X$ is locally bounded. The trick is to leverage the result for locally bounded processes by subtracting the big jump from $X$. Assume without loss of generality that $X_{0}$ and defined $J_{t}=\sum_{s \leq t} \Delta X_{s} 1_{\left|\Delta s_{\mathrm{s}}\right| \geq 1}$. Then $X=X-J$ is an adopted, cadlag locally bounded process. We will show 
that theorem 1.4 for $X$ implies NFLVR + LI for $X$, so that we may apply theorem 1.3 to $X$. Then since $J$ is finite variation, this will then imply $X$ is a classical semimartingale .

Suppose $\phi^{n} \in X$ are such that $\left\|\phi^{n}\right\|_{u} \rightarrow 0$ and $\left\|\left(\phi^{n} \cdot X\right)^{-}\right\|_{u} \rightarrow 0$. We need to prove that $\left(\phi^{n} \cdot X\right)_{t} \rightarrow 0$ in probability . First we will show that $\left\|\left(\phi^{n} \cdot X\right)^{-}\right\|_{u} \rightarrow 0$.

$$
\begin{aligned}
& \sup _{0 \leq t \leq T}\left(\phi^{n} \cdot X\right)_{t}^{-} \\
& \leq \sup _{0 \leq t \leq T}\left(\phi^{n} \cdot X\right)_{t}^{-}+\sup _{0 \leq t \leq T}\left|\left(\phi^{n} \cdot J\right)_{t}\right| \\
& \leq \sup _{0 \leq t \leq T}\left(\phi^{n} \cdot X\right)_{t}^{-}+\left(\left\|\phi^{n}\right\|_{\infty} \cdot T V(J)\right)_{T} \rightarrow 0
\end{aligned}
$$

by the assumptions on $\phi^{n}$

By $(1),\left(\phi^{n} \cdot X\right)_{T} \rightarrow 0$ in probability. Since $\left(\phi^{n} \cdot J\right)_{T} \rightarrow 0$ in probability, we conclude that

$$
\left(\phi^{n} \cdot X\right)_{T}=\left(\phi^{n} \cdot J\right)_{T}-\left(\phi^{n} \cdot J\right)_{T} \rightarrow 0
$$

in probability. Therefore $X$ satisfies NFLVR + LI.

\section{REFERENCES}

[1] F. Delbaen and W. Schachermayer, "A General Version of the Fundamental Theorem of Asset Pricing," Mathematische Annalen, Vol. 300 No. 3, 1994, pp.463-520. doi:10.1007/BF01450498

[2] P. E. Protter, "Stochastic Integration and Differential Equation,” 2nd Edition, Springer-Verlag, Berlin, 2004.
[3] M. Beiglblock, W. Schachermayer and B. Veliyev, "A Direct Proof of the Bichteler-Dellacherie Theorem and Connections to Arbitrage," 2010.

[4] M. Loewenstein and G. A. Willard, "Local Martingales, Arbitrage, and Viability Free Snacks and Cheap Thrills," Economic Theory, Vol. 16, No. 1, 2000, pp.135-161. doi: $10.1007 / \mathrm{s} 001990050330$

[5] E. Platen, "Arbitrage in Continueous Complete Markets," Advances in Applied Probability, Vol. 34, No. 3, 2002, pp.540-558. doi:10.1239/aap/1033662165

[6] R. Fernholz, I. Karatzas and C. Kardaras, "Diversity and Relative Arbitrage in Equity Markets," Finance and Stochastics, Vol. 9, No. 1, 2005, pp.1-27. doi:10.1007/s00780-004-0129-4

[7] C. Kardaras and E. Platen, "On the Semimartingale Property of Discounted Asset-Price Processes," Stochastic Processes and Their Applications, Vol. 121, 2011, pp. 2678-2691.

[8] F. Delbaen and W. Schachermayer, "Arbitrage and Free Lunch with Bounded Risk for Unbounded Continuous Processes," Mathematical Finance, Vol. 4, No. 4, 1994, pp. 343-348. doi:10.1111/j.1467-9965.1994.tb00063.x

[9] D. Williams, "Probability with Martingales," Cambridge University Press, Cambridge, 1991. doi:10.1017/CBO9780511813658

[10] I. Karatzas and S. E. Shrve, "Brownian Motion and Stochastic Calculus of Graduate Text in Mathematics," 2nd Edition, Springer-Verlag, Berlin, 1991.

[11] J. Komlos, "A generalization of problem of Steinhaus," Acta Mathematica Academiae Scientiarum Hungarica, Vol. 18, No. 1-2, 1967, pp. 217-229. doi:10.1007/BF02020976 